\title{
Distinguishing KBO from Asteroid Occultations in TAOS
}

\author{
Claudia Lemme \\ Institute of Astronomy, National Central University, 32054 Chung-Li, \\ Taiwan
}

Chyng-Lan Liang

Department of Statistics, University of California, Berkeley, USA

\begin{abstract}
The goal of the Taiwan-America Occultation Survey (TAOS) project is to estimate directly the number of Kuiper Belt Objects (KBOs) by measuring the rate of stellar occultations. Occultations of distant stars may also be caused by asteroids and this article describes how both types of occultations can be distinguished on the basis of their shadow ground speed on Earth and the observing angle.
\end{abstract}

\section{Introduction}

A flattened annulus beyond the orbit of Neptune, named the "Kuiper belt", is thought to be the source of short-period comets. Containing the most pristine material, this region provides valuable diagnostics for the early stages of Solar System formation. As the point of origin of the short-period comets, it is also relevant for calculations on the number of Earth-crossing bodies and thus for the frequency of biological extinction events. Since observational success was first achieved in 1992, more than 300 Kuiper belt objects (KBOs) have been found. So far however, direct imaging has been sensitive only to the larger members of the belt, those with diameters greater than around $50 \mathrm{~km}$. The extent, size distribution and total mass of the Kuiper belt are all still unknown, and are of especial interest considering the opposing theories that abound (e.g. Jiang, I.G., this volume). The Taiwan-America Occultation Survey (TAOS, see King, S.K., this volume) will be able to measure the number of these objects down to $\sim 3$ $\mathrm{km}$, by their chance occultations of background stars. Several telescopes will operate in coincidence mode to distinguish real occultations from false events, that result from noise or occultations by terrestrial objects. However, a fraction of the real occultations is expected to be produced by asteroids, and not by KBOs. To distinguish these, we consider the speed of the shadow ground track on Earth.

\section{Results}

The speed of the shadow ground track is basically given by the relative velocity with respect to Earth, perpendicular to the line of sight of an object at distance 


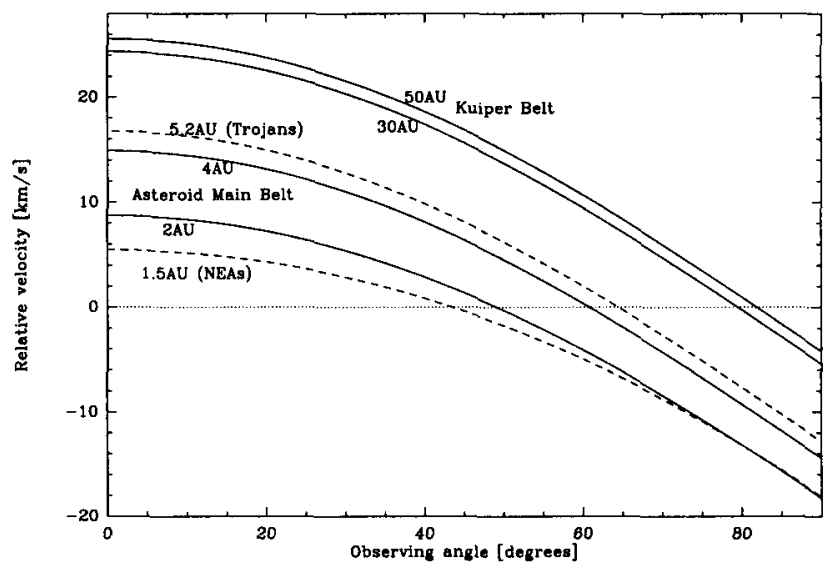

Figure 1. Relative velocity (corresponds to the speed of the shadow ground track on Earth) as function of observing angle for the Kuiper belt between 30 and $50 \mathrm{AU}$ and the asteroid main belt between 2 and $4 \mathrm{AU}$. In addition, the relations for the Trojan asteroids and a typical Near-Earth asteroid are marked by dashed lines.

$r$ and observing angle $\phi$. For circular, plane orbits this is given by:

$$
R V(r, \phi)=v_{e} \cos \phi-v_{e} \sqrt{\left(\frac{r_{e}}{r}\right)\left(1-\left(\frac{r_{e}}{r}\right)^{2} \sin ^{2} \phi\right)}
$$

where $r_{e}$ is the heliocentric distance of Earth and $v_{e}$ the orbital velocity of Earth. The observing angle is measured clockwise from opposition (when the Earth is located between the object (KBO/asteroid) and the Sun). Figure 1 reveals:

1. For observing angles $\phi<42^{\circ}$, both $\mathrm{KBOs}$ and asteroids move retrograde (RV $>0$, this corresponds to an East $\rightarrow$ West movement of the shadow on Earth). 2. Each asteroid has an "angle of quadrature" $(\mathrm{RV}=0)$ between $\phi \sim 42^{\circ}$ and $65^{\circ}$. The asteroid's speed is 0 (or close to 0 ) at this angle.

3 . For $\phi>65^{\circ}$, all the asteroids move prograde.

4. For $80^{\circ}<\phi<82^{\circ} \mathrm{KBOs}$ reach their angle of quadrature.

5. For $\phi>82^{\circ}$ both KBOs and asteroids move prograde.

But most importantly for distinguishing the two groups: for any observing angle, there is always a difference in the shadow ground speed. Around opposition this is $\sim 13 \mathrm{~km} / \mathrm{s}$ (and at least $7 \mathrm{~km} / \mathrm{s}$ between the outermost Trojans and the innermost KBOs). For a $\phi$ between $60^{\circ}$ and $80^{\circ}$ the absolute speed for both can be similar, but the asteroids move prograde and the KBOs retrograde. The difference in speed affects occultation duration and arrival time at the different telescopes, and the arrival time allows us to determine whether the occultation was caused by an asteroid or a KBO. Note that the distinction can not be made by a single telescope alone and we need telescopes separated far enough apart from each other in East-West direction. We expect to detect about 1 asteroid occultation every 10 or $100 \mathrm{KBO}$ occultations; the range reflects the uncertainty in the total number of asteroids and KBOs. 